\title{
Functional Magnetic Resonance Imaging
}

Todd Parrish, Ph.D.

Director of the Center for Advanced MRI

Director of MR Neuroimaging Research

Associate Professor

Department of Radiology

Northwestern University

toddp@northwestern.edu

The field of MR brain mapping is made possible by exploiting properties of cerebral hemodynamics subsequent to neuronal activity. With the onset of neuronal activity there is an associated change in cerebral metabolism, blood flow, blood volume and oxygen consumption [1-5]. The complex physiologic interplay of these parameters is not well understood. However, current research efforts are aimed at understanding these physiologic parameters and would certainly improve the methodology and localization of functional magnetic resonance imaging.

The first functional brain images were the work of Belliveau et al. [6]. The detection of blood flow changes associated with different brain states was accomplished with the use of an exogenous contrast agent. These experiments required two different contrast injections during different mental states, flashing light and darkness. The difference in cerebral blood flow was evident by the contrast (flow) related changes. The drawback to this method was the required use of contrast and therefore, the inability to do several experiments in a single subject.

A different type of MR hemodynamic-based method, which did not require exogenous contrast, was developed independently by Kwong et al. and Ogawa [7-9]. In Kwong's experiments the MR signal contrast was derived from either the $\mathrm{T} 1$ shortening due to changes in blood flow or the differences in oxygenation. Kwong showed in both a visual and motor task that it was possible to measure brain activation. Ogawa, on the other hand, had been investigating the MR signal properties associated with oxygenated and deoxygenated blood [10]. The focus of this work was to use blood as an endogenous contrast agent. As the fraction of oxygenated to deoxygenated blood changes so does the MR signal. This effect has been called BOLD, blood oxygenation level dependent, imaging. This is by far the method of choice in most functional imaging labs studying primary sensory and motor areas as well as higher levels of cognition such as attention, language, and memory.

How is it possible to measure brain activation from blood oxygenation changes in the blood? A generally assumed model of the physiology of brain activation is given below.

1. Cortical activation occurs. There is a local demand for energy in the form of oxygen or glycogen. Figure 1-a.

2. A hemodynamic delay occurs on the order of 4-8 seconds. Figure 1-b.

3. There is an increase in the capillary perfusion (blood flow and/or volume) in the region to deliver the metabolic products. This occurs with minimal (or larger) increase in $\mathrm{O}_{2}$ consumption.

4. With the increase in perfusion, there is an increase in oxygenated blood. The oxy/deoxy ratio has increased. Therefore, the MR signal has increased. Figure 1-c

5. Once the demand for blood supply stops (no more activity), the perfusion is reduced. There is a hemodynamic lag for this process as well. In addition, a well documented undershoot follows the activation. It is believed to be a function of the vascular system. Figure 1-d

6. After 10-90 seconds, the oxy/deoxy ratio returns to normal values and the MR signal decreases to normal resting levels. Figure 1-e 


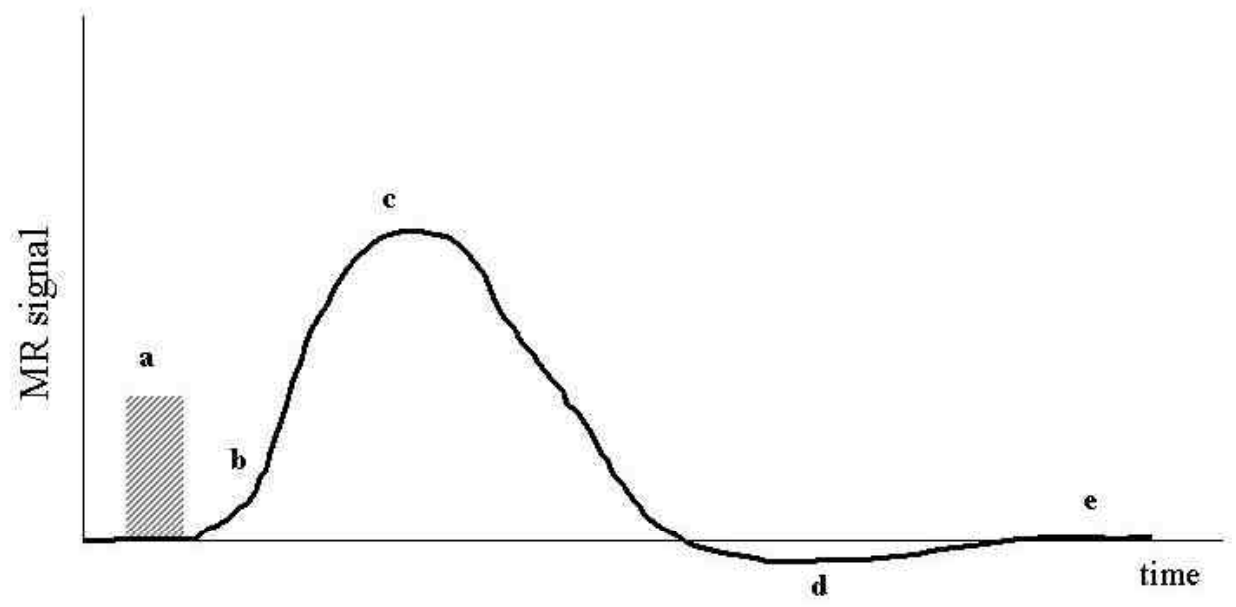

Figure 1. Time course of MR signal in response to a short stimulus (a, hatched region).

This simplified version of the physiologic changes during neuronal activation is still under debate. Other experimental methods such as optical imaging, PET imaging and MR spectroscopy have documented blood flow changes, blood volume changes, blood oxygenation and oxy/deoxy changes [2, 5, 6,11-13]. These studies are just starting to unravel the complex puzzle of the physiology of activation.

One of the most fundamental concepts in functional magnetic resonance imaging is the realization that the measured signal is indirectly related to function. The MR signal is sensitive to changes in the oxy/deoxy ratio, which in turn is sensitive to a number of physiologic parameters including CBV, CBF, and $\mathrm{O}_{2}$ consumption. Furthermore, there is a hemodynamic delay of 4-8 seconds because the measured response is related to blood flow [7,14-18]. A study by D'Esposito has shown that the hemodynamic response varies dramatically from subject to subject [1]. However, the response in a single subject across days is similar. The ability to detect the hemodynamic response in different brain regions is very difficult and fraught with experimental complications such paradigm design, cognitive load and temporal resolution. The mean time to peak in D'Esposito's study was calculated to be 4.7 seconds. This delay is significantly longer than the tens to hundreds of milliseconds in electrically based methods. Thus, the temporal resolution of fMRI falls somewhere between EEG and PET. However, fMRI has the added advantage of high resolution, tomographic slices, which can be obtained in any orientation.

\section{The BOLD experiment}

The goal of the functional magnetic resonance imaging experiment is to modulate the activity of the brain in conjunction with a means to identify the brain regions that change signal intensity in sync with the paradigm. Generally, a boxcar scheme is used to define the experiment. In this case, the active and rest states are alternated with a known frequency, each having a known duration. In turn, the blood flow in the area of interest will change in accordance with the activity, see figure 2 . It is important to understand that these signal changes are relative to the background task. Any activation inferred needs to be described relative to the cognitive background level. Note the lag in the MR signal (blood flow response), in figure 2. An alternate method for experimental design is the use of single events spaced in time. Initially these vents were spaced such that the hemodynamic response would not overlap with the previous event. These experiments were very long, non-naturalistic for the subject and had very low power. Then it was shown by several groups that it is possible to treat this as a linear system and allow the events to be spaced closer together [15-16]. In this case, the events can be spaced 2-3 seconds apart and the individual responses be resolved. The advent of rapid event-related fMRI has greatly improved the field and expanded our knowledge about the brain. For example, it is possible to investigate what neuronal network is responsible for proper encoding of memory by reviewing the brain responses to correct memory. But what is more interesting is to investigate the brain regions that are (not) involved in the incorrect encoding of memories to identify the breakdown in the process. 


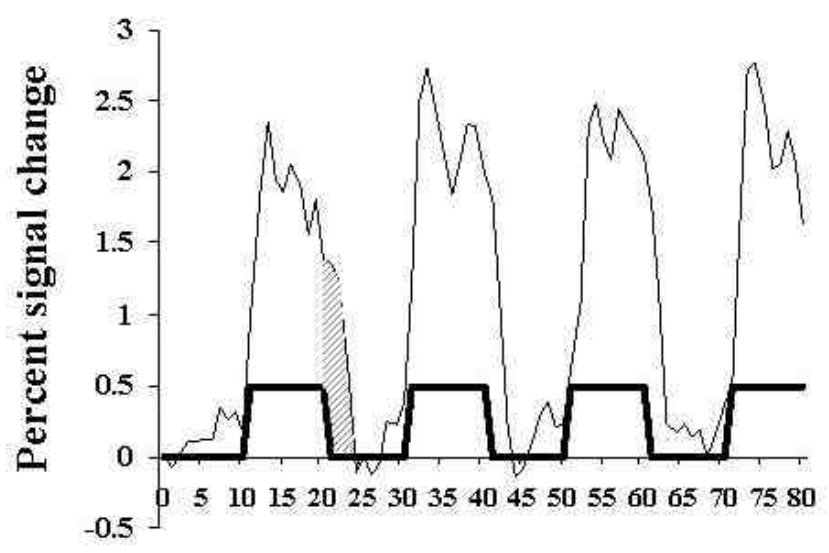

Volume number

Figure 2. Schematic of a typical fMRI experiment showing the hemodynamic delay to the stimulus.

\section{$\underline{\text { Practical Fundamentals of Functional MRI (fMRI) }}$}

Functional MRI is a field of Neuroimaging that detects small changes in blood oxygenation in cortical regions that are synchronized with the presentation of a specific paradigm. During the paradigm presentation, the scanner collects many slices ( 24-36 slices) using echo planar imaging in a short amount of time, $\sim 2$ seconds. The result is a time series of volume data (4D). Since the magnitude of the change is quite small, $\sim 1 \%$, several pre-processing steps improve the detection of the activation induced signal changes. After the pre-processing has been applied, a statistical test or model can be used to determine the significance of the changes that occur. Finally, the statistically significant regions, "blobs", are coregistered to a high-resolution dataset for display and interpretation.

Pre-processing

In all fMRI experiments there is a dummy period where the MR signal is allowed to reach a steady-state. Typically the first 10-12 seconds (5-6 dummy scans if TR=2s) of scanning is discarded. It is important to make sure that the appropriate amount of time is allotted in the paradigm design for the signal to reach steady state. Otherwise, there would be a timing error and the resultant activations would not reflect activation due to the particular portion of the paradigm.

Once the appropriate data has been retained, one must perform motion correction of the data. The goal of motion correction is to make sure that the volumes of data acquired are spatially aligned so that when a voxel is projected over time it represents the same brain tissue over time, see figure 3 .

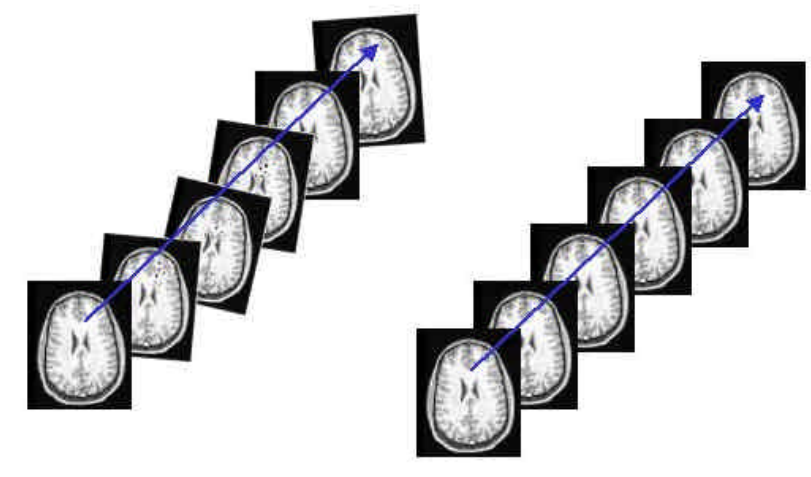

Figure 3

Typically, the user identifies a reference volume at a specific time point (default is the first image in the series). This volume of data is compared to the next in the time series. An iterative process of shifting and rotating the new volume to match the reference volume is performed. The goal is to minimize the 
difference between the two datasets. Once this has been accomplished the resultant shift and rotation is applied to that volume and is used as the starting point for the next volume. This continues until all the time points have been corrected. A plot of the shifts and rotations is informative about how well the subject behaved and to determine if there are any activation induced motion artifacts, see figure 4 . The motion correction algorithm can correct for sub-voxel movements but has difficulty in large shifts. This is due to the MR signal dependence on the RF pulsing history. Therefore, slow drifts in the data are acceptable but large $(0.5 \mathrm{~mm}$ or larger) volume-to-volume jumps are not. In the event that these jumps exist, one has to throw out the data or try to salvage portions that do not exhibit motion.

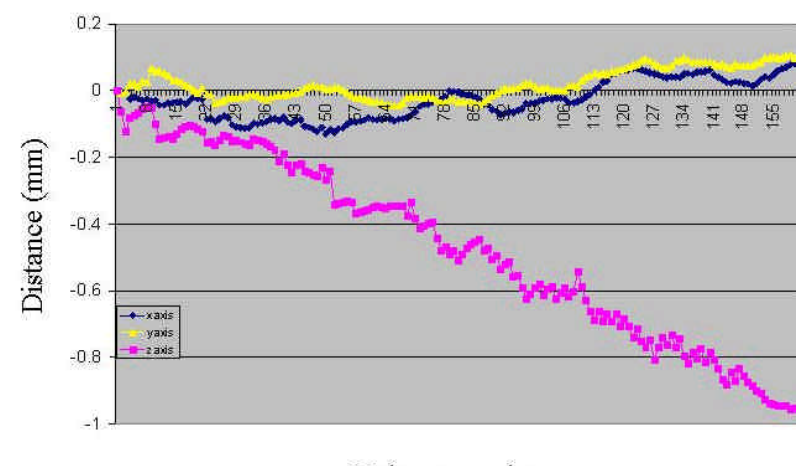

Volume number

Figure 4

A controversial area of pre-processing for fMRI has been the issue of spatial and temporal smoothing. The benefit of smoothing is an increase in the apparent signal to noise. There is a dramatic effect with a small amount of smoothing, however, the downside of smoothing is that the localization of the activation is compromised. The range of spatial smoothing is anywhere from $5 \mathrm{~mm}$ up to $12 \mathrm{~mm}$ depending on the goal of the experiment. Obviously, with the larger smoothing kernel the goal of the research is to investigate larger network issues within the brain. Larger smoothing kernels are also used when data are normalized to a standard template to improve the transformation process by removing individual differences. The issue of temporal smoothing is somewhat less of an issue as the hemodynamic response is sluggish compared to the TR used in the fMRI experiment. The effect of smoothing in the time dimension is to reduce the physiologic fluctuations that occur and help reduced volume-to-volume signal changes that are a result of the imaging hardware.

The statistical analysis of functional data has always been a hot bed of controversy. It starts with the fact that the data are not independent samples as many statistics assume. There is an auto-correlation issue with the formation of MR data especially if one smoothes the data (i.e. mix signals in time and space from different voxels). Furthermore, there is the issue of multiple comparisons and how does one correct for these. In a typical dataset, there are $131072(64 \times 64 \times 32)$ voxels, which can be reduced based on some intensity threshold but the number is still quite large. What does a confidence level mean with such a large number? For example a confidence level of $95 \%(?=0.05)$ means that you are willing to accept a $5 \%$ error. In the above example that would mean 6554 voxels would be false positives. How do you adjust the statistic to account for all the comparisons being made? The Bonferroni correction is a very conservative approach, which works well with a small number of comparisons, however it can be used as a guide. The correction considers each comparison separately but with a fraction of the desired confidence level. In the example above, the desired significance level is 0.05 and the number of comparisons is 131072 . The Bonferroni correction would impose a significance level of $0.000000381(0.05 / 131072)$ on each comparison to ensure that the overall significance level for the entire dataset was maintained at 0.05 . In practice this is much to conservative. Each functional imaging software package has developed methods to address this issue. For example, SPM (http://www.fil.ion.ucl.ac.uk/spm/) invokes random Gaussian field theory to determine levels of significance and the thresholds that should be applied. Still other packages have utilized Monte Carlo simulations to develop a model of the noise of random data to guide the significance level and the thresholds that are applied to the data. The bottom line is that the theory 
used to determine the level of significance applied to the data has to be known by the user in order to interpret the data responsibly.

Another factor that goes into the threshold calculation used on the statistical maps is the amount of connectivity imposed. There should be more significance put on 5 voxels that are suprathreshold and connected than 5 individual islands of activation, where the latter has more potential to be noise. Now this is a tricky situation especially when there is a large amount of smoothing employed. You can imagine that if you take a single voxel with a large amount of activation and smooth it to cover a large area, the surrounding voxels will benefit. This could boost these voxels above the threshold and connect them so that they meet the cluster threshold. The areas of activation could be enlarged. Again it is important that the investigator understand how the data are preprocessed and what impact that will have on the generation of the statistical maps.

Finally a statistic with an appropriate threshold needs to be applied to the data. In some functional imaging software packages a model of the paradigm is constructed and used to fit the data. For example, it is known when the active and rest periods occur. This can be combined with a hemodynamic response to simulate the expected response from the vascular based signal. Accounting for motion correlated signal changes, physiologic induced variations, global signal changes over time and even difficulty of the task presented can further enhance the model. The more information supplied to the model the better the estimate of the activation induced signal changes. However, if the model is designed with poor information, it is possible to increase the error resulting in poor activation detection. The opposite end of the spectrum in functional brain imaging is to not assume too much about the signal response. This is advantageous because it can apply to any type of experiment but the residual signal has a large amount of variance. This increased variance may reduce the detection of the activation induced signal change or it may have a structure that can be used to improve the model. Nevertheless, there is a large spectrum of functional imaging analysis packages that have been developed by individual labs to accomplish the level of modeling or statistics that are required to attack a specific issue. It is important to understand the assumptions and basis of the software package that is being used to generate the statistical maps.

Once the statistical map has been created, it needs to be displayed. The first step in this process is to make sure that the high resolution anatomic data $(2 \mathrm{D}$ or $3 \mathrm{D})$ is coregistered with the functional data. The coregistration step ensures that the underlying anatomy is the source of the activation induced signal change. Next the functional data needs to be interpolated to the resolution of the anatomic data. Typical dimensions of the fMRI data are 64x64 and the anatomic data are 256x256. Bilinear interpolation can be used to increase the matrix size of the fMRI data. This operation takes into account the neighboring data values when creating the data to fill-in between the original data. Sinc interpolation is a better method but requires more computational effort since it incorporates more of the data when determining a voxel value. A larger problem is what to do in the slice dimension. Usually, the fMRI data has a thickness of 3-4mm whereas the anatomic data is typically $1 \mathrm{~mm}$ thick. Simple duplication of the statistical over $4 \mathrm{~mm}$ of the brain may not represent the true activation location or continuity. The use of trilinear or sinc interpolation is preferred to ensure that the continuity of the functional activation is preserved.

With the functional significance maps overlaid on the anatomic data, how does one demonstrate activation? This is accomplished by manipulating the look up table (LUT) for the image. Typical MR images are displayed in grayscale with black=minimum value (1) and white=maximum value (256). The user controls the window width (contrast) of pixel values displayed and the center level (brightness) of the window to create the desired image contrast. In the case of fMRI data, the LUT is modified such that the lower portion (1-192) corresponds to the typical grayscale LUT for the anatomic image. The upper portion (193-256) corresponds to the area where the functional information is stored, see figure 5. 


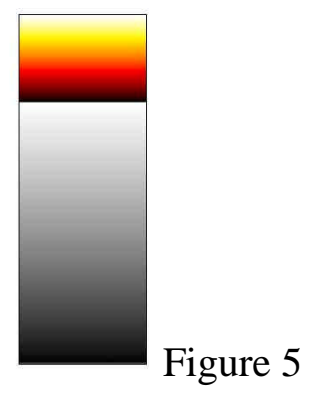

In most cases this means that a color LUT is used for the upper band. Typically the hot metal colormap is used such that regions that just meet significance are red/orange, higher levels of significance are yellow and the most significant are white. The cool metal colormap is used for negative activations. The 3D dataset with the modified LUT can be viewed with any 3D package to allow you to slice away the brain to expose the underlying activations or render the brain to visualize the activation patterns on the surface of the brain, see figure 6 .

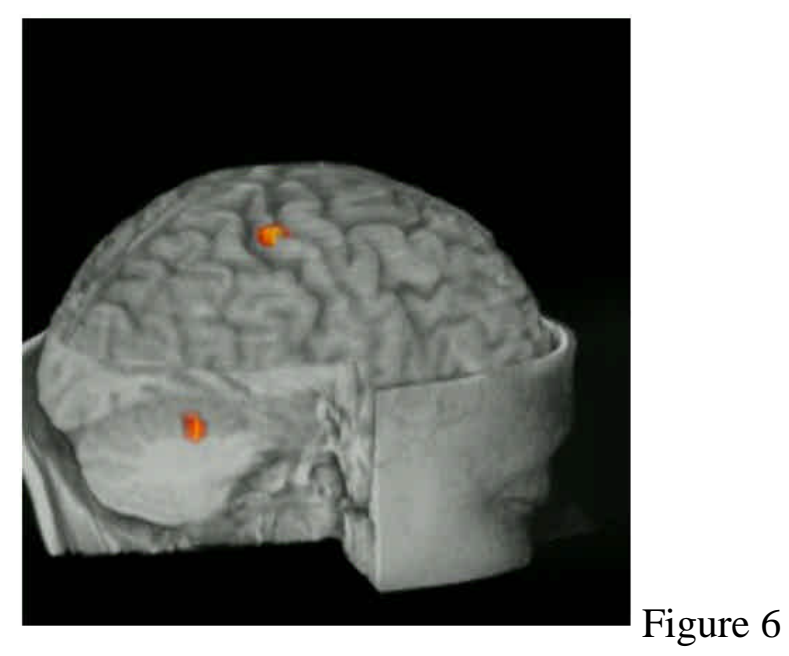

\section{References:}

1. Belliveau, J.W., Rosen, B.R., Kantor, H.L., Rzedzian, R.R., et al., Functional cerebral imaging by susceptibility-contrast NMR. Magnetic Resonance in Medicine, 1990. 14(3): p. 538-46.

2. Fox, P.T. and Raichle, M.E., Focal physiological uncoupling of cerebral blood flow and oxidative metabolism during somatosensory stimulation in human subjects. Proc. Natl. Acad. Sci. U.S.A., 1986. 83(4): p. 1140-1144.

3. Ingvar, D.H., Functional landscapes of the dominant hemisphere. Brian Res., 1976. 107: p. 181-197.

4. Phelps, M.E., Kuhl, D.E., and Mazziota, J.C., Metabolic mapping of the brain's response to visual stimulation: studies in human. Science, 1981. 211: p. 1445-1448.

5. Prichard, J., Rothman, D., Novotny, E., Petroff, O., et al., Lactate rise detected by 1 H NMR in human visual cortex during physiologic stimulation. Proc. Natl. Acad. Sci. U.S.A., 1991. 88: p. 5829-5831.

6. Belliveau, J.W., Kennedy, D.N., Jr., McKinstry, R.C., Buchbinder, B.R., et al., Functional mapping of the human visual cortex by magnetic resonance imaging. Science, 1991. 254(5032): p. 716-9.

7. Kwong, K.K., Belliveau, J.W., Chesler, D.A., Goldberg, I.E., et al., Dynamic magnetic resonance imaging of human brain activit. during primary sensory stimulation. Proceedings of the National Academy of Sciences of the United States of America, 1992. 89(12): p. 5675-9.

8. Ogawa, S. and Lee, T.M., Magnetic resonance imaging of blood vessels at high fields: in vivo and in vitro measurements and imag, simulation. Magnetic Resonance in Medicine, 1990. 16(1): p. 9-18.

9. Ogawa, S., Lee, T.M., Kay, A.R., and Tank, D.W., Brain magnetic resonance imaging with contrast dependent on blood oxygenation. Proceedings of the National Academy of Sciences of the United States of America, 1990. 87(24): p. 9868-72.

10. Ogawa, S., Lee, T.M., Nayak, A.S., and Glynn, P., Oxygenation-sensitive contrast in magnetic resonance image of rodent brain a high magnetic fields. Magnetic Resonance in Medicine, 1990. 14(1): p. 68-78. 
11. Fox, P.T., Burton, H., and Raichle, M.E., Mapping human somatosensory cortex with positron emission tomography. J Neurosurg 1987. 67: p. 34-43.

12. Fox, P.T., Raichle, M.E., Mintun, M.A., and Dence, C., Nonoxidative glucose consumption during focal physiologic neural activity Science, 1988. 241: p. 462-464.

13. Malonek, D. and Grinvald, A., Interactions between electrical activity and cortical microcirculation revealed by imaging spectroscopy: implications for functional brain mapping. Science, 1996. 272(5261): p. 551-4.

14. Bandettini, P.A., Jesmanowicz, A., Wong, E.C., and Hyde, J.S., Processing strategies for time-course data sets in functional MRI 0 the human brain. Magnetic Resonance in Medicine, 1993. 30(2): p. 161-73.

15. Boynton, G.M., Engel, S.A., Glover, G.H., and Heeger, D.J., Linear systems analysis of functional magnetic resonance imaging i। human V1. Journal of Neuroscience, 1996. 16(13): p. 4207-21.

16. D'Esposito, M., Zarahn, E., and Aguirre, G.K., Event-related functional MRI: Implications for cognitive psychology. Psychologica Bulletin, 1999. 125(1). p. 155-64.

17. Hu, X., Le, T.H., and Ugurbil, K., Evaluation of the early response in fMRI in individual subjects using short stimulus duration. Magnetic Resonance in Medicine, 1997. 37(6): p. 877-84.

18. Kim, S.G., Richter, W., and Ugurbil, K., Limitations of temporal resolution in functional MRI. Magnetic Resonance in Medicine, 1997. 37(4): p. 631-6.

19. Aguirre, G.K., Zarahn, E., and D'Esposito, M., The variability of human, BOLD hemodynamic responses. NeuroImage, 1998. 8(4) p.360-9. 